\title{
NEW ELECTRONICALLY-TUNABLE OSCILLATOR CIRCUIT USING ONLY TWO OTAs
}

\author{
MUHAMMAD TAHER ABUELMA'ATTI* \\ and MUHAMMAD HAROON KHAN \\ King Fahd University of Petroleum and Minerals, \\ Box 203, Dahran 31261, Saudi Arabia
}

(Received 28 December 1996; In final form 13 March 1997)

\begin{abstract}
New oscillator circuit using two operational transconductance amplifiers and grounded capacitors is presented. The oscillator circuit enjoys independent control of the frequency and the condition of oscillation. Experimental results confirming the presented theory are included.
\end{abstract}

Keywords: Oscillators; operational transconductance amplifiers

\section{INTRODUCTION}

The potentials of the operational transconductance amplifier (OTA) as an integrated circuit building block alongwith its applications in generating all finite linear circuits were introduced early by Bialko et al. [1]. The OTAs have many attractive features. For example, they require just a few or even no resistors for their internal circuitry, provide highly linear electronic tunability of its transfer gain and have more reliable high-frequency performance.

Over the past few years, a number of schemes have been developed for realizing OTA-based sinusoidal RC-oscillators [2-8]. Most of these circuits use two OTAs in combination with a number of grounded and floating capacitors and resistors. From a practical point

*Corresponding author. 
of view an oscillator circuit is attractive if (1) a single-element control of the frequency of oscillation is possible without disturbing the condition of oscillation and (2) a single element control of the condition of oscillation is possible without disturbing the frequency of oscillation. Most of the oscillator circuits available in [2-8] do not enjoy these two attractive features simultaneously.

The major intention of this paper is to present a new OTA-based oscillator circuit. The proposed circuit uses two OTAs, two grounded capacitors, a buffer and one floating resistor and enjoys independent control of the frequency and the condition of oscillation.

\section{PROPOSED CIRCUITS}

Consider the oscillator structure shown in Figure 1. Assuming an ideal OTA, routine analysis of the circuit yields its characteristic equation given by

$$
s^{2}+\alpha_{0} S+\beta_{0}=0
$$

where

$$
\alpha_{0}=1 / C_{2} R_{2}+1 / C_{2} R_{1}
$$

and

$$
\beta_{0}=g_{m 1 / C_{2} C_{3} R_{1}}
$$

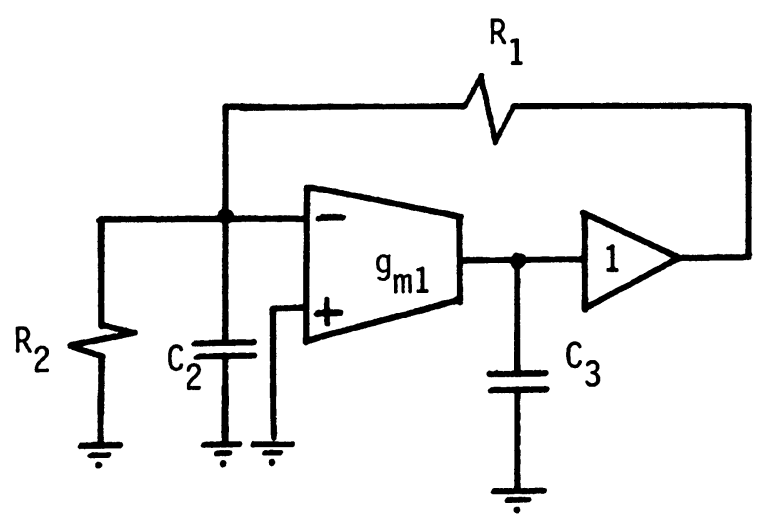

FIGURE 1 Proposed Oscillator Circuit. 
Equation (1) generates a pair of complex conjugate poles with a small positive real part. These poles, $\sigma_{p} \pm j \omega_{p}$, are given by

$$
\sigma_{p}=-\alpha_{0} / 2
$$

and

$$
\omega_{p}=\left(4 \beta_{0}-\alpha_{0}^{2}\right)^{1 / 2} / 2
$$

Obviously, if $\sigma_{p}>0, \omega_{p}$ will be the frequency of oscillation of the circuit. From (2) and (4) one can see that for $\sigma_{p}>0$, it is required that

$$
R_{2}<0
$$

and

$$
R_{1}>\left|R_{2}\right|
$$

Also, from (4) and (5) one can see that if $\sigma_{p}=0_{+}$, then the frequency of oscillation of the circuit will be

$$
\omega_{0}=\beta_{0}^{1 / 2}
$$

From (6) - (8) one can see that obtaining oscillation from the circuit of Figure 1 is feasible subject to realising $R_{2}$ as a negative resistance. Realising a negative resistance is straightforward using an operational transconductance amplifier [9]. Figure 2 shows a modified version of the proposed circuit of Figure 1 with $R_{2}$ realised using an OTA. Using (2)-(8) it is easy to show that the frequency of oscillation and the condition of oscillation of the circuit of Figure 2 will be

$$
\omega_{0}^{2}=g_{m 1} / C_{2} C_{3} R_{1}
$$

and

$$
g_{m 2} R_{1}=1
$$

From (9) and (10) one can see that the condition of oscillation and the frequency of oscillation are totally uncoupled. Thus, while the 


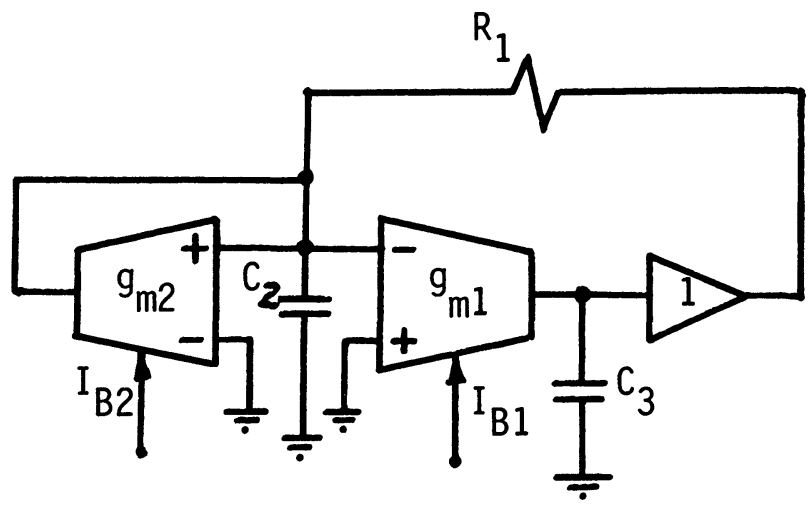

FIGURE 2 Modified version of Figure 1 with $R_{2}$ realised as a negative resistance= $-1 / g_{m 2}$.

frequency of oscillation can be adjusted by tuning $g_{m 1}$ without disturbing the condition of oscillation, the condition of oscillation can be adjusted by tuning $g_{m 2}$ without disturbing the frequency of oscillation. And since the transconductance gain of the OTA $\left(g_{m i}\right)$ is linearly proportional to the amplifier bias current $I_{A B C i}$ of the OTA then the frequency of oscillation and the condition of oscillation can be independently adjusted by changing the amplifier bias currents. Moreover if these currents are obtained from the output of digital-toanalog converters (DACs) then digital programming of the frequency of oscillation and the condition of oscillation is feasible.

\section{EXPERIMENTAL RESULTS}

The circuit of Figure 2 was tested using the LM13600 OTA. This chip contains two OTAs and two buffers. Thus the circuit of Figure 2 requires only one IC chip. The results obtained are shown in Figure 3. Good quality sinusoidal oscillations were obtained. From Figure 3 one can see that the measured and calculated results are in good agreement. 


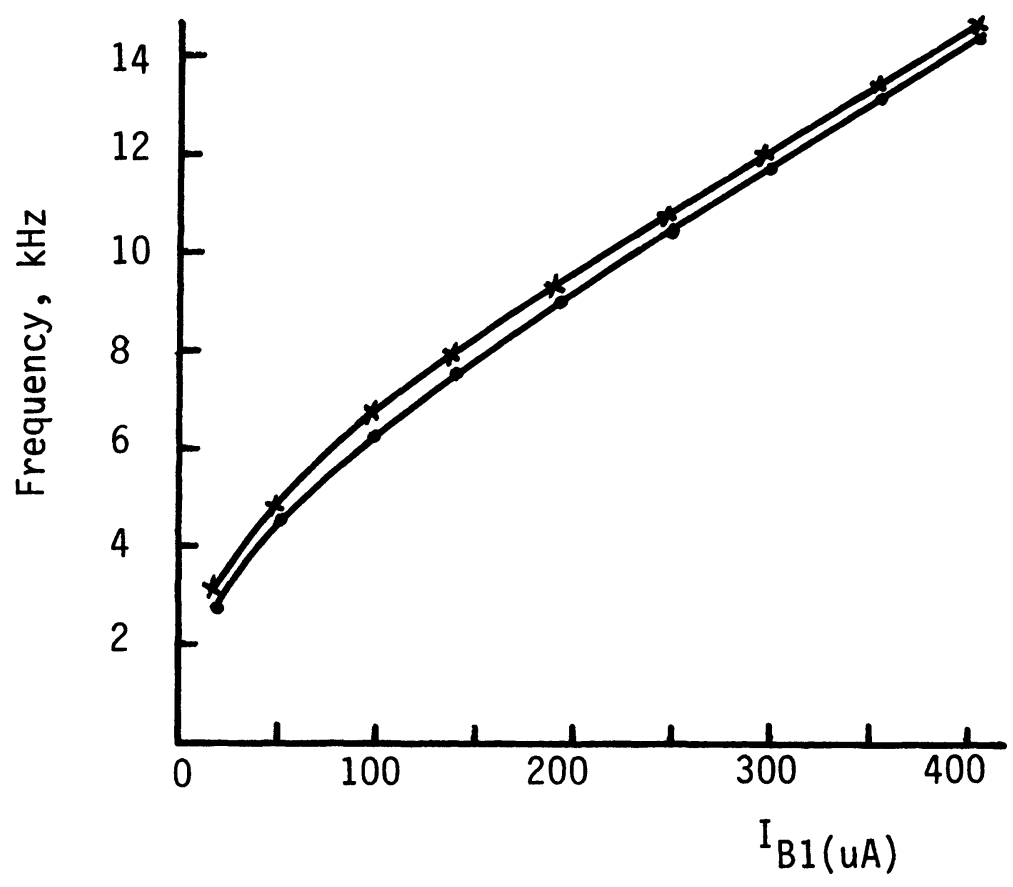

FIGURE 3 Measured and calculated results obtained from Figure 2 with: $C_{1}=C_{3}=10$ nf $R_{1}=10 k, I B_{2}=4 \mu \mathrm{A} \longrightarrow$ Measured $-\longrightarrow-$ Calculated.

\section{CONCLUSION}

In this paper a new OTA-based RC oscillator circuit has been presented. The circuit uses two OTAs, one buffer, one resistor and two grounded capacitors. Thus it can be realised using one IC chip (the LM13600). The circuit enjoys independent control of the frequency and the condition of oscillation.

It is worth mentioning here that fully integrable, entirely OTAbased oscillator can be obtained, at the cost of two extra OTAs, by simulating the resistor $R_{1}$ by two OTAs. Alternatively, from an IC implementation viewpoint $R_{1}$ could be a resistor either external to the chip or can be a part of a feedback electronic circuit used to stabilise (or control) the amplitude of oscillation. 


\section{References}

[1] Bialko, M. and Newcomb, R. W. (1971). Generation of all finite linear circuits using the DVCCS, IEEE Transactions on Circuit Theory, CT-18, 733-736.

[2] Abuelma'atti, M. T. and Almaskatti, R. H. (1989). Identification of two-amplifiers OTA-based sinusoidal oscillators, Proceedings International Conference on Signals and Systems, Brighton, England, 4, 249-260.

[3] Abuelma'atti, M. T. and Almaskatti, R. H. (1990). Novel two-amplifier OTA-based sinusoidal RC-oscillator circuits, Proceedings International Conference on Signals and Systems, Al-Ain, U.A.E., 2, 39-48.

[4] Saha, A. R., Nandi, R. and Nandi, S. (1983). Integrable tunable sinusoidal oscillator using DVCCS, Electronic Letters, 19, 745-746.

[5] Hoyle, J. and Sinencio, S. E. (1984). Sinusoidal quadrature OTA oscillators, 27th IEEE Midwest Symposium on Circuits and Systems, pp. 59-62.

[6] Nandi, R. (1982). Novel variable-frequency oscillator with single resistor control using DVCCS/DVCVS, Electronics Letters, 18, 144-145.

[7] Ahmed, M. T. and Khan, I. A. (1984). Realization of integrable electronically tunable oscillators with operational transconductance amplifiers, IEEE International Conference on Consumer and Industrial Electronics and Applications, 20-21.

[8] Abuelma'atti, M. T. and Almaskatti, R. H. (1989). New active-RC sinusoidal oscillators using the operational transconductance amplifier, Proceedings International Conference on Signals and Systems, Miami, Florida, U.S.A. pp. 3-8.

[9] Geiger, R. L. and Sanchez-Sinencio, E. (1985). Active filter design using OTAs: A tutorial, IEEE Circuits and Devices Magazine, 1, 20-32. 

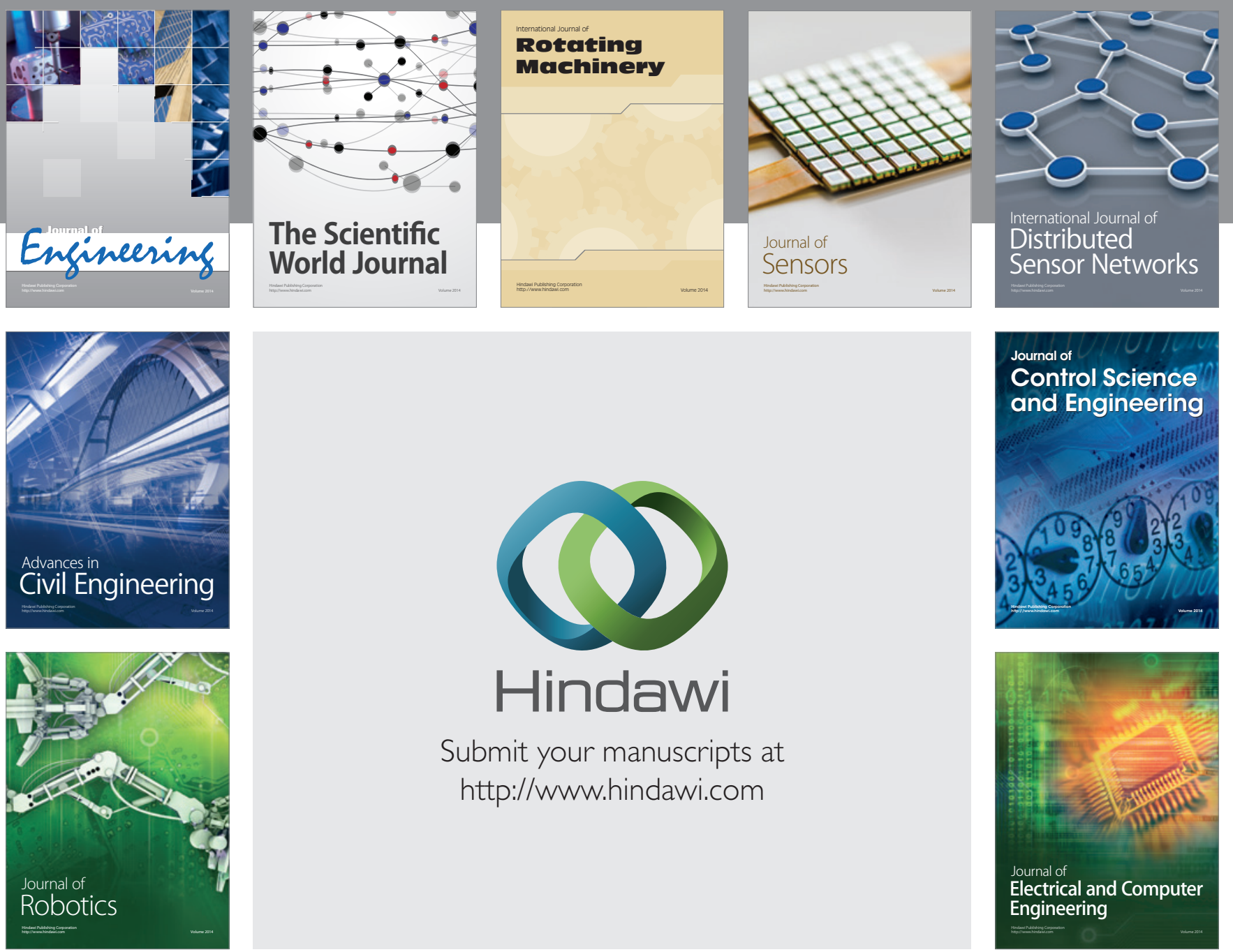

Submit your manuscripts at

http://www.hindawi.com
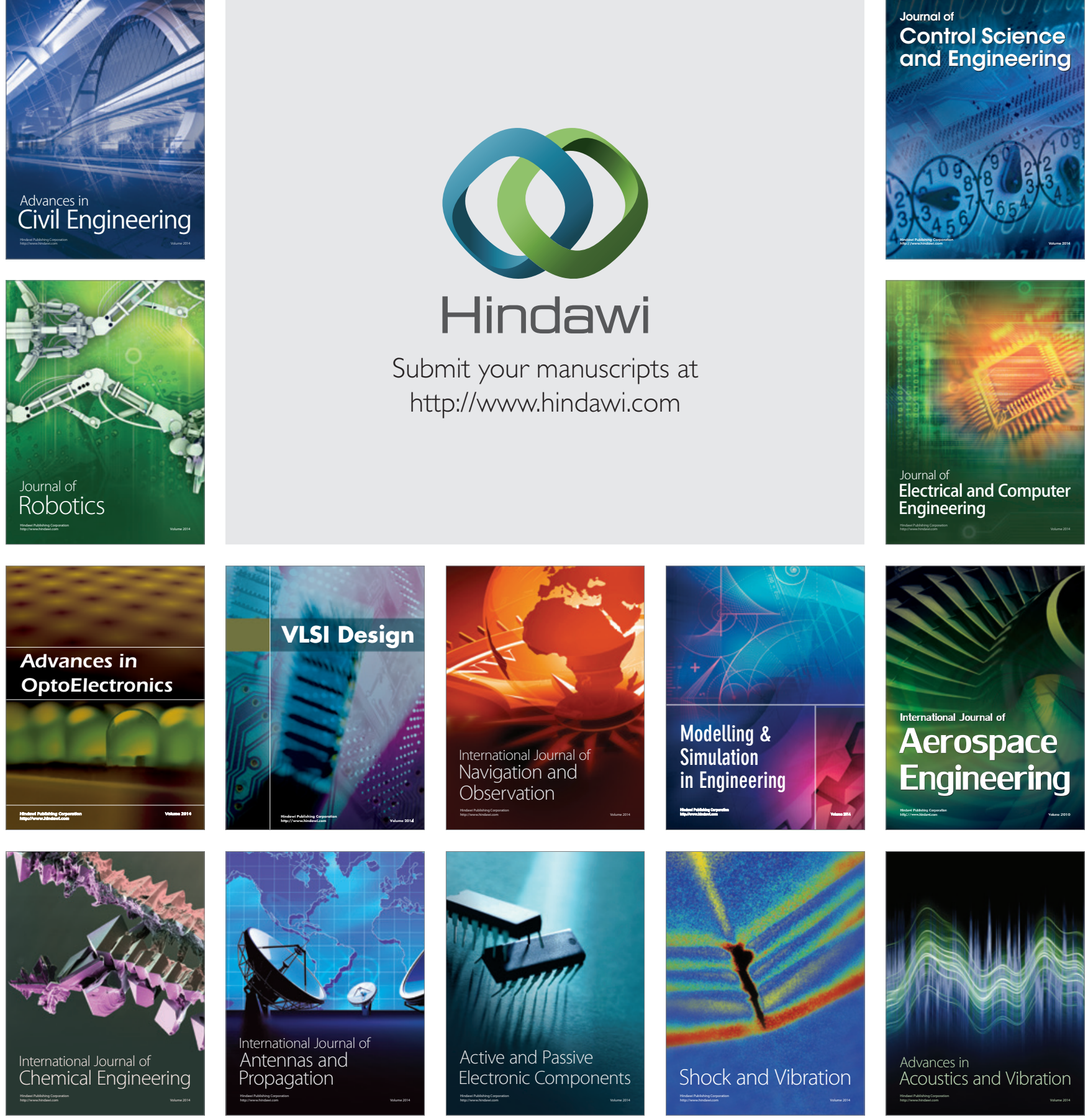